CALIFORNIA PATH PROGRAM

INSTITUTE OF TRANSPORTATION STUDIES

UNIVERSITY OF CALIFORNIA, BERKELEY

\title{
Analysis of Channel Access Schemes for Model-based Estimation over Multi-access Networks
}

\section{Ching-Ling Huang Raja Sengupta}

\section{California PATH Research Report \\ UCB-ITS-PRR-2009-6}

This work was performed as part of the California PATH Program of the University of California, in cooperation with the State of California Business, Transportation, and Housing Agency, Department of Transportation, and the United States Department of Transportation, Federal Highway Administration.

The contents of this report reflect the views of the authors who are responsible for the facts and the accuracy of the data presented herein. The contents do not necessarily reflect the official views or policies of the State of California. This report does not constitute a standard, specification, or regulation.

Final Report for 5214

January 2009

ISSN 1055-1425 


\section{Contents}

1 EXECUTIVE SUMMARY 3

2 INTRODUCTION 4

3 RELATED WORK 5

4 Problem Formulation $\quad 6$

4.1 NCS over Multi-Access Channel . . . . . . . . . . . . 6

4.2 Model-Based Estimation . . . . . . . . . . . . . . . 7

4.3 Performance Metric: MSE . . . . . . . . . . . . . . . 8

5 Probabilistic Channel Access 9

5.1 Case $|a|=1 \ldots \ldots \ldots$. . . . . . . . . . . . . . . . . . . . . . . . 9

5.2 Case $0<|a|<1 \ldots \ldots \ldots \ldots . \ldots \ldots$

5.3 Case $1<|a|<\infty \ldots \ldots \ldots \ldots . \ldots \ldots$

6 Deterministic Channel Access 13

6.1 Case $|a|=1 \ldots \ldots \ldots \ldots$. . . . . . . . . . . . . . . . . . . . . . . 13

6.2 Case $0<|a|<1 \ldots \ldots \ldots \ldots$. . . . . . . . . . 14

6.3 Case $1<|a|<\infty \ldots \ldots \ldots \ldots$

7 Hybrid Channel Access $\quad \mathbf{1 5}$

7.1 Case $|a|=1 \ldots \ldots \ldots \ldots$. . . . . . . . . . . . . . 16

7.2 Case $0<|a|<1 \ldots \ldots \ldots \ldots$. . . . . . . . . . . . . . . . . . . . . . . . . . 17

7.3 Case $1<|a|<\infty \ldots \ldots \ldots \ldots$

$\begin{array}{lll}8 & \text { Summary and Future Work } & 18\end{array}$ 


\title{
Analysis of Channel Access Schemes for Model-based Estimation over Multi-access Networks
}

\author{
Ching-Ling Huang and Raja Sengupta*
}

January 8, 2009

\begin{abstract}
This report investigates the performance of model-based estimation over multi-access networks and emphasizes on the estimation MSE (mean squared error) while using different channel access schemes: probabilistic (random access), deterministic (round-robin scheduling), and combined (grouped channel access). We propose a mathematical framework for estimation over a simple multi-access MAC protocol, the slotted ALOHA network. Estimation MSE, its asymptotic behavior and stability condition are derived for different channel access methods. Our quantitative discussion can provide guidelines to design the communication logic for those vehicular control systems built on top of multi-access networks.
\end{abstract}

keywords: vehicular control, model-based estimation, multi-access channel, slotted ALOHA

*This work was supported by Caltrans through UCB PATH T.O. 5214, "ITS Band Roadside to Vehicle Communications in a Highway Setting - Protocol Layer," and has been presented in IEEE International Symposium on Intelligent Control, San Antonio, TX, Sept. 2008.

${ }^{\dagger}$ The authors are with the Civil System Group, Department of Civil and Environmental Engineering, University of California, Berkeley, CA 94720. Contact: clhuang@path.berkeley.edu 


\section{EXECUTIVE SUMMARY}

This document is an interim report submitted in partial fulfillment of the requirements for UCB-PATH TO 5214 titled ITS Band Roadside to Vehicle Communications in a Highway Setting. In particular it addresses Task 1.2 Develop DSRC Medium Access Control Layer.

The most challenging application planned for deployment over DSRC is Cooperative Active Safety (CAS). In the CAS concept, vehicles will send information such as GPS position, speed, and heading to neighboring vehicles over a DSRC channel. The receiving vehicle can use the incoming messages to detect if the sending vehicle is a threat. If so it may warn its driver. Such message are expected to be about 200 bytes long and may have to be transmitted as far as 300 meters, this being the stopping distance of a vehicle traveling at freeway speeds, when it limits itself to brake pressures comfortable for the passengers. Studies such as those undertaken by the NHTSA sponsored Vehicle Safety Communications Consortium, have suggested these messages might have to be broadcast by a vehicle as frequently as every 100 milliseconds. Finally, several competent simulation studies in the literature show that at these operating conditions, the DSRC channel becomes heavily congested, leading to high loss rates. More than 30\% of the messages may fail to reach their intended target. The DSRC medium access control layer needs to be designed to overcome this problem.

This report describes mathematical idea useful for DSRC medium access control design. Vehicles can be viewed as dynamical systems, and abstractly, the DSRC channel is to be designed for each dynamical system to efficiently estimate the state of the others. Viewed this way, one finds the recent literature on Networked Control Systems, produced by the control community, gives insight into how the DSRC channel might be designed. This report starts with ideas in the control literature and then advances them to address the following architectural question arising in DSRC design.

Some researcher reports, for example, one written by us for the Cooperative Vehicle Highway Automation System program, have examined the use of roadside radios to schedule transmission by vehicle radios. The aim of scheduling is to divide time into slots and ensure that only one vehicle transmits in a slot. This avoids the collision problem, i.e., a situation in which two or more vehicles transmit in a slot. When this happens, the signals of the multiple transmitters collide at any receiver within range of both, and the receiver fails to receive every one of the transmitted messages. The slot 
is wasted. A roadside radio can be used to reduce this wastage. The benefit comes at the cost of installing a roadside infrastructure to help the vehicle to vehicle communication. The alternative is for this communication to occur by unassisted vehicle to vehicle cooperation, i.e., in the ad-hoc networking style. The principal contribution of the report is to quantify the relative benefit of the assisted case over the unassisted case. The comparison measure is mean square estimation error. The ad-hoc case is modeled as slotted ALOHA. The comparison is computed by developing an analytical model.

\section{INTRODUCTION}

In recent years, many control applications of distributed systems are built on top of networks for information exchange, such as AHS (Automated Highway Systems) and UAV (Unmanned Aerial Vehicles). Those systems are called Networked Control Systems (NCSs) in which sensors, actuators, controllers communicate through a data network. In NCSs, estimation is known to be a critical step, and its accuracy directly affects the control performance.

For example, imagine that there is a group of intelligent vehicles equipped with wireless transceivers, traveling on the highway. Each vehicle can estimate neighboring vehicles' position and speed information, via a shared channel, and uses this information to facilitate certain safety applications, such as collaborative collision avoidance and the electronic brake light system.

For control over lossy channel, a decade of research $[3,4,5,6,7]$ shows that the right approach is probably to insert a model-based estimator in between controller and the sensor. If the channel does not deliver sensor measurements on time, the estimator uses its model to provide the controller with its best estimate of the state. When measurements are successfully received, the estimator uses them to improve its estimate for the state of the remote system. The controller is always fed by the estimator.

In NCSs, the question of how to use minimum bit rate to control/stabilize a system through feedback was first introduced in $[8,9]$. Towards better modeling of today's digital networks, i.e. state information is transferred via packets instead of bit streams, the minimum packet rate problem was investigated in $[10,11,13,14]$. In existing literature, estimation problem is usually formulated as the sender-receiver pair with one-to-one channel scenario. Channel losses are usually assumed to be independent events. However, this assumption is not realistic for most multi-access networks since a collision 
happens when more than one node transmits data at the same time.

To better understand the performance of estimation over a multi-access network, we propose a framework and analyze MSE (mean squared error) of model-based estimation on top of slotted ALOHA [1], a simple multi-access network. Specifically, we compare the estimation performance of three channel access designs: 1) probabilistic design that utilizes fully random access to the channel; 2) deterministic design in which nodes are perfectly scheduled to transmit; and 3) hybrid design that is combined from probabilistic and deterministic channel access.

Among three communication methods, probabilistic channel access is easier to implement in a decentralized fashion but would inevitably incur collisions. On the other hand, deterministic scheme can avoid collisions by scheduling but it may require out-of-band signaling for coordination or at least consensus among nodes. In our analysis, asymptotic behavior and stability conditions for estimation MSE using those channel access schemes are also derived.

The contribution of this work is its quantitative discussion on model-based estimation in a multi-access channel setting. The organization of this report is the following: Section II states related work, and Section III describes our problem formulation. Section IV, V, and VI are devoted to the analysis of probabilistic, deterministic, and hybrid channel access designs respectively. Section VII concludes this report with a summary and future work.

\section{RELATED WORK}

Stability constraints for partial observations are investigated in $[11,12]$. In the problem formulation [11], raw sensor measurements are transmitted to remote estimators and the channel drops packets according to i.i.d. Bernoulli trials. On the receiver side, intermittent observations are processed with a time-varying Kalman filter. The threshold of channel loss probability to stabilize estimation error is also derived. In [15], multiple description (MD) codes, a type of network source codes, are designed to compensate for packetdropping and communication delay for Kalman filtering.

In [13], the sender keeps track of successfully transmitted state information as well as performs estimation of its local process, which is assumed to reach the same estimation results by the receiver. Long-term average cost problem is formulated for this scenario. Optimal controlled communication 
policy is derived with the cost function defined as weighted summation of estimation MSE and packet rate. The optimal decision to broadcast status update at each moment turns out to be decided by the current estimation error. The optimal communication policy is also proved to be a threshold policy, in which a region is defined for the estimation error. When the error exceeds this region/threshold, a broadcast is triggered to update the state information on remote estimators.

In [14], authors suggest that each node should process raw measurements with a Kalman filter before sending them out. The stability condition of such pre-processing is compared with [11]. The minimum packet rate to stabilize the estimation error to the stochastic moments is given in $[10,14]$ for uncontrolled communication logic triggered by one fixed-rate Poisson process. Controlled communication logic is also proposed based on the Doubly Stochastic Poisson Process (DSPP) [16] to trigger the transmission. At each moment, jump intensity of the DSPP is a function of current estimation error. This error-dependent policy is proven to effectively keep all finite moments of estimation errors and the communication rate bounded.

Classical information theory [2] deals with the encoding of long sequences of data and thus inherently needs to tolerate long latency. However, due to the interactivity of real-time control, the state information to be communicated is not known ahead in time and it is used to control the very process being encoded. A new metric for evaluating channels in terms of reliability, called Anytime Capacity, is defined on a sense of reliable transmission in [17] and extended to multi-access channels in [18]. A summary of recent advances in NCSs can be found in [19].

\section{Problem Formulation}

In this section, we describe our mathematical framework of model-based estimation on top of multi-access channel as foundation for analysis in following three sections.

\subsection{NCS over Multi-Access Channel}

Suppose there are $n$ nodes sharing a slotted ALOHA network, $n=2,3, \ldots$, and each node contains a discrete-time LTI scalar process (1), a transmission control logic, and a bank of synchronized model-based estimators (see Fig. 


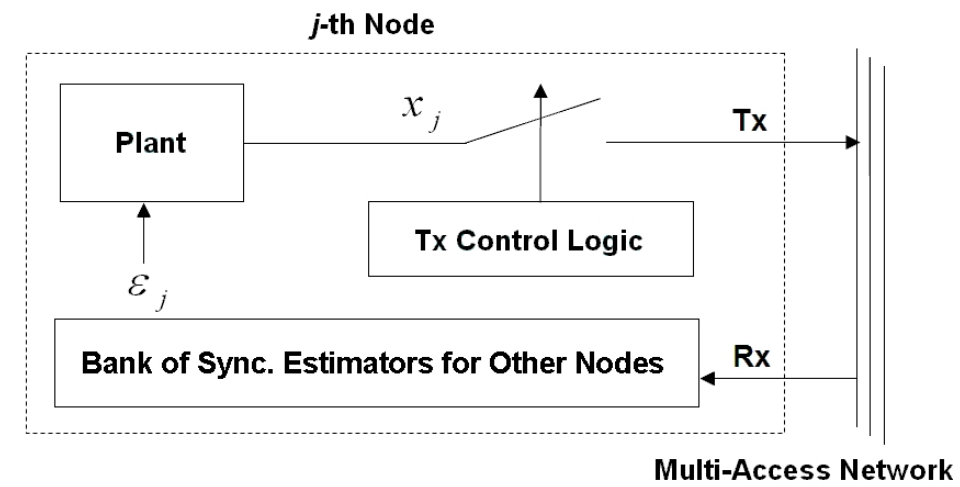

Figure 1: Node internal structure of analyzed NCS estimation problem. Each node contains an LTI plant, a transmission control logic, and a bank of model-based estimators for other nodes. The channel access scheme used for transmission control will be specified and analyzed later.

1). Each node will try to estimate the state on other nodes by received information from the shared channel.

In our setting, the dynamics of those spatially distributed processes are assumed to be completely decoupled. For notation convenience, let node $j \in\{1,2, \ldots, n\}$ represent the sender in following derivation,

$$
x_{j}(t)=a_{j} x_{j}(t-1)+\varepsilon_{j}(t-1)
$$

where $x_{j}(t)$ is the scalar state of node $j$ and $\varepsilon_{j}(t)$ is $i . i . d$. zero-mean noise process with finite variance $\sigma_{j}^{2}$, and $t$ is time index, $t \in N$.

Similar to the minimum packet rate formulation in $[13,14]$, at each time index, the true state (a real number with acceptable distortion) of the sender $j$ could be transferred to a receiver $i \neq j, i \in\{1,2, \ldots, n\}$, via the shared and possibly lossy network. The transmission control logic decides whether to broadcast its own state information to others at each moment. Furthermore, the transmission time of state information, i.e. one slot length, is assumed to be the same as one discrete step of the process (1).

\subsection{Model-Based Estimation}

Let $\tilde{x}_{i j}(t)$ be the estimated state of sender $j$ at receiver $i$. And, this estimated state is the expectation conditioned on all the previous received information 
from the lossy channel,

$$
\tilde{x}_{i j}(t)=E\left[x_{j} \mid Y_{i}^{1}, Y_{i}^{2}, \ldots, Y_{i}^{t-1}\right]
$$

where $Y_{i}^{t}, t=1,2, \ldots$, is the received information at moment $t$ at the receiver $i$.

When channel is idle or has a collision at $t, Y_{i}^{t}=\emptyset$. Otherwise, $Y_{i}^{t}=x_{s}(t)$ from a certain successful sender $s \neq i, s \in\{1,2, \ldots, n\}$. In our formulation, channel loss is only due to collisions. Neither fade effect nor hidden terminal problem is modeled for the multi-access channel. Therefore, each node is assumed to get the same copy of information from the channel.

The model-based estimator at receiver $i$ switches between following two modes:

- If no information regarding node $j$ is received at $t-1$, i.e. $Y_{i}^{t-1} \neq$ $x_{j}(t-1)$, use previous estimate $\tilde{x}_{i j}(t-1)$ and the known model (1) to carry on,

$$
\tilde{x}_{i j}(t)=a_{j} \times \tilde{x}_{i j}(t-1)
$$

- Else if state information of $j$ is received at $t-1$, i.e. $Y_{i}^{t-1}=x_{j}(t-1)$, use it to reset estimation error,

$$
\tilde{x}_{i j}(t)=a_{j} \times x_{j}(t-1)
$$

Note that $\tilde{x}_{i j}(t)$ in $(3),(4)$ is the optimal estimate, in MMSE sense, because noise process in (1) has zero mean.

\subsection{Performance Metric: MSE}

In general cases when $\sigma_{j}^{2}>0$ and $a_{j} \neq 0$, the performance metric is MSE (mean squared error) of the estimation process at the receiver side. For the estimator on node $i$, if an update is only received $k$ steps before, $k=$ $1,2, \ldots t-1$, the best estimate of $x_{j}$ is

$$
\tilde{x}_{i j}(t)=a_{j}^{k} x_{j}(t-k)
$$

Assuming latest measurement arrives at receiver side at the $t-k$ moment, now let $\varphi_{i j}(t)$ be the MSE of above estimation process, i.e. node $j$ tries to estimate node $i$ based on received information. $\varphi_{i j}(t)$ is given by definition,

$$
\varphi_{i j}(t)=E\left[\left(x_{j}(t)-\tilde{x}_{i j}(t)\right)^{2}\right]
$$


By substituting $\tilde{x}_{i j}(t)$ derived in (5), conditioned on elapsed time step $k$ since receiving an update from $j,(6)$ becomes

$$
\varphi_{i j}(t)=E\left[E\left[\left(x_{j}(t)-a_{j}^{k} x_{j}(t-k)\right)^{2} \mid k<t\right]\right]
$$

The inner part of (7) can be expressed as

$$
x_{j}(t)-a_{j}^{k} x_{j}(t-k)=\sum_{l=1}^{k} a_{j}^{k-l} \varepsilon_{j}(t-(k-l+1))
$$

where $\varepsilon_{j}(t)$ is the noise process at moment $t$ for node $j$.

Since the noise process $\varepsilon_{j}(t)$ are i.i.d. zero-mean random variables with finite variance $\sigma_{j}^{2}$, then (7), i.e. node $i$ 's estimation MSE for state $j$, can be organized as

$$
\varphi_{i j}(t)=E\left[\sum_{l=1}^{k} a_{j}^{2(k-l)} \mid k<t\right] \times \sigma_{j}^{2}
$$

where $E\left[\sum_{l=1}^{k} a_{j}^{2(k-l)} \mid k<t\right]$ can be further specified once the channel access design and the probability distribution of inter-arrival time $k$ is known. In the following three sections, we will analyze the estimation performance of three different channel access schemes, namely probabilistic, deterministic, and hybrid methods, based on (9). Throughout this report, we further assume the process on each node is identical, i.e. $a_{j}=a$ and $\sigma_{j}=\sigma$ for all $j$.

\section{Probabilistic Channel Access}

In this section, we consider a purely random access scheme. Let each node broadcast its own state information with a fixed probability $p_{j}$ at each time slot. To derive the optimal uniform probability $p^{*}$ for all nodes, i.e. $p_{j}=$ $p^{*}$ for all $j$, we need to consider several cases. Specifically, we denote the estimation MSE, defined in (6), while using the probabilistic method as $\varphi_{j}^{P}$. (We drop subscript $i$ since all nodes can get the same copy of information from the channel and achieve the same estimation for node $j$.)

\subsection{Case $|a|=1$}

Consider process (1) with bounded noise variance $\sigma^{2}>0$ and $|a|=1$. First, let's focus on the case when $a=1$,

$$
x_{j}(t)=x_{j}(t-1)+\varepsilon_{j}(t-1)
$$


which can be seen as a one-dimensional random walk with each step decided by the noise process. Without any broadcast of state information, i.e. $p_{j}=0$ for all $j$, the best estimate of the state is given by

$$
\tilde{x}_{j}(t)=E\left[x_{j}(0)+\sum_{l=0}^{t-1} \varepsilon_{j}(l)\right]=x_{j}(0)
$$

if initial state $x_{j}(0)$ is given. However, estimation MSE of this process without any broadcast, denoted as $\bar{\varphi}_{j}(t)$, is given by

$$
\bar{\varphi}_{j}(t)=E\left[\left(x_{j}(t)-\tilde{x}_{j}(t)\right)^{2}\right]=E\left[\sum_{l=0}^{t-1} \varepsilon_{j}^{2}(l)\right]=t \times \sigma^{2}
$$

Thus, as $t \rightarrow \infty$, the estimation MSE will go unbounded, i.e. $\bar{\varphi}_{j}(t) \rightarrow \infty$, which shows the necessity for each node to broadcast state information to eliminate the estimation error, i.e. $0<p_{j} \leq 1$.

To find the optimal broadcast probability $p_{j}=p^{*}$ for all $j$, let's focus on the estimation MSE for node $j$ since all nodes are identical. In this case, (9) can specified as

$$
\varphi_{j}^{P}(t)=E\left[E\left[\sum_{l=t-k}^{t-1} \varepsilon_{j}^{2}(l) \mid k<t\right]\right]=E[k \mid k<t] \times \sigma^{2}
$$

Considering $t \rightarrow \infty$, (13) implies the optimal probability $p^{*}$ is the minimizer of mean inter-arrival time $k$ of state information of $j$ delivered to node $i$, i.e.

$$
p^{*}=\underset{0<p \leq 1}{\arg \min } E[k]
$$

Since all nodes share a slotted ALOHA network, the probability for receiver $i$ to get an update from node $j$ successfully, received at $k$ slots ago, can be written as

$$
P_{i j}(k=K)=p(1-p)^{n-1} \times\left[1-p(1-p)^{n-1}\right]^{K-1}
$$

where $K=1,2, \ldots, t-1$. With (15), $E[k \mid k<t]$ can be given by definition,

$$
E[k \mid k<t]=\sum_{k=1}^{t-1} k \times p(1-p)^{n-1} \times\left[1-p(1-p)^{n-1}\right]^{k-1} .
$$


which can be organized into a closed form if $t \rightarrow \infty$,

$$
E[k]=\left(p(1-p)^{n-1}\right)^{-1}
$$

Therefore, to find the stationary solution $p^{*},(14)$ can be rewritten as

$$
p^{*}=\underset{0<p \leq 1}{\arg \min }\left(p(1-p)^{n-1}\right)^{-1}
$$

The optimal (stationary) solution to above problem is the well-known result for slotted ALOHA [1]:

$$
p^{*}=\frac{1}{n}
$$

which maximizes the per node throughput $p(1-p)^{n-1}$. For the case $a=-1$, (17) can also be shown to be optimal.

\subsection{Case $0<|a|<1$}

Now consider the process (1) with bounded noise variance $\sigma^{2}>0$ and $0<$ $|a|<1$. From (9), the general form of estimation MSE can be specified as

$$
\varphi_{j}^{P}(t)=\sigma^{2}\left(\frac{1}{1-a^{2}}-\frac{E\left[a^{2 k} \mid k<t\right]}{1-a^{2}}\right)
$$

Since $a^{2}<1$, to minimize MSE, we focus on finding $p_{j}=p^{*}$, for all $j$, to maximize $E\left[a^{2 k} \mid k<t\right]$ in (18). Now considering $t \rightarrow \infty$, we want to find $p^{*}$ to maximize $E\left[a^{2 k}\right]$,

$$
p^{*}=\underset{0<p \leq 1}{\arg \max } E\left[a^{2 k}\right] .
$$

where the stationary formulation as $t \rightarrow \infty$,

$$
E\left[a^{2 k}\right]=\sum_{k=1}^{\infty} P_{i j}(k) \times a^{2 k}
$$

Plugging (15) into (20), we get

$$
E\left[a^{2 k}\right]=p(1-p)^{n-1} \times \sum_{k=1}^{\infty}\left[1-p(1-p)^{n-1}\right]^{k-1} \times a^{2 k} .
$$


Because $\left[1-p(1-p)^{n-1}\right] \times a^{2}<1$, above can be organized to a closed form,

$$
E\left[a^{2 k}\right]=\frac{p(1-p)^{n-1} \times a^{2}}{1-\left[1-p(1-p)^{n-1}\right] \times a^{2}}
$$

Now let $q=p(1-p)^{n-1}$, which denotes the per node steady-state throughput in slotted ALOHA system. From the throughput analysis in [1], we know

$$
0<q \leq \frac{1}{n}\left(1-\frac{1}{n}\right)^{n-1}<1
$$

Thus (21) can be expressed as

$$
E\left[a^{2 k}\right]=\frac{q \times a^{2}}{1-(1-q) \times a^{2}}
$$

Let $h_{a}(q)$ be a function of $q$ with fixed $a$ as its parameter,

$$
h_{a}(q)=\left(1+\frac{1-a^{2}}{q a^{2}}\right)^{-1}=E\left[a^{2 k}\right]
$$

The fact that $0<a^{2}<1$ together with (22) implies that $h_{a}(q)$ is monotone increasing as $q$ increases. With (24), now the maximization problem (19) is equivalent to

$$
q^{*}=\underset{0<q \leq \frac{1}{n}\left(1-\frac{1}{n}\right)^{n-1}}{\arg \max } h_{a}(q)
$$

where optimal solution exists at $q^{*}=\frac{1}{n}\left(1-\frac{1}{n}\right)^{n-1}$, which is the maximum per node throughput achieved in slotted ALOHA network. Therefore, the optimal broadcast probability is given by (17) for the case $0<|a|<1$.

\subsection{Case $1<|a|<\infty$}

Now consider the process (1) with bounded noise variance $\sigma^{2}>0$ but $1<$ $|a|<\infty$. Since $1<a^{2}<\infty$, from (9) we can get the general form of MSE as (18). To get bounded MSE as $t \rightarrow \infty$, i.e. $\lim _{t \rightarrow \infty} \varphi_{j}^{P}(t)<\infty, E\left[a^{2 k}\right]$ must be bounded. Similar to previous case, let $q=p(1-p)^{n-1}$, we have

$$
E\left[a^{2 k}\right]=q \times \sum_{k=1}^{\infty}(1-q)^{k-1} \times a^{2 k}
$$


To have $E\left[a^{2 k}\right]$ bounded, $q$ and $a$ must satisfy below condition,

$$
(1-q) \times a^{2}<1
$$

Here we get a relationship between the per node steady-state throughput $q$ and the parameter $a$ if the estimation MSE is bounded for $t \rightarrow \infty$. Together with (22), to have $\lim _{t \rightarrow \infty} \varphi_{j}^{P}(t)$ bounded, $a$ has to satisfy below condition:

$$
|a|<\left(1-\frac{1}{n}\left(1-\frac{1}{n}\right)^{n-1}\right)^{-\frac{1}{2}}
$$

where $n$ is the number of nodes. This upper bound monotonically converges down to 1 when $n$ is sufficiently large. If $a$ satisfies (27), optimal broadcast probability can be shown to be (17). Otherwise, when $|a| \geq\left(1-\frac{1}{n}(1-\right.$ $\left.\left.\frac{1}{n}\right)^{n-1}\right)^{-\frac{1}{2}}$, the estimation process cannot have bounded MSE, as $t \rightarrow \infty$, by using a fixed broadcast probability for all nodes.

\section{Deterministic Channel Access}

Probabilistic design explored in Section IV can be viewed as the completely randomized channel access. For multi-access channel, one can also choose TDMA (Time Division Multiple Access) scheme for each node to broadcast state information without collisions in the shared channel.

In this report, deterministic design refers to the round-robin scheduling that fairly serves each node. All nodes are assumed to have this TDMA scheduling knowledge. At each moment, there will be only one node scheduled to broadcast its own state information and thus there is no collision (loss). In the following analysis, we denote the estimation MSE, defined in (6), while using round-robin scheme as $\varphi_{j}^{D}$.

\subsection{Case $|a|=1$}

For the process (1) with bounded noise variance $\sigma^{2}>0$ and $|a|=1$. Similarly, (9) can be specified as the form in (13). Now, with a deterministic communication scheduling, and the estimation MSE is bounded by

$$
\sigma^{2} \leq \varphi_{j}^{D}(t) \leq n \times \sigma^{2}
$$

depending on the moment $t$ and scheduled communication instants for node $j$. 
Recall that, from (13), (15) and (17), the steady-state minimum estimation MSE for probabilistic design, denoted as $\varphi_{j}^{P}(t)^{*}$, is given by

$$
\varphi_{j}^{P}(t)^{*}=n\left(1-\frac{1}{n}\right)^{1-n} \times \sigma^{2}
$$

When $n$ is sufficiently large, (29) can be approximated by

$$
\varphi_{j}^{P}(t)^{*} \rightarrow n e \times \sigma^{2}
$$

which reveals that $\varphi_{j}^{P}(t)^{*}$ increases with the number of nodes $n$. By comparing (28) and (29), we can see that,

$$
\varphi_{j}^{P}(t)^{*}>\varphi_{j}^{D}(t)
$$

which shows that, in this simple case $|a|=1$, using a deterministic channel access can achieve lower estimation MSE than that of probabilistic design as $t \rightarrow \infty$.

\subsection{Case $0<|a|<1$}

Now consider the process (1) with bounded noise variance $\sigma^{2}>0$ and $0<$ $|a|<1$. From (9) we can get the general form of estimation MSE,

$$
\varphi_{j}^{D}(t)=\frac{1-a^{2 L_{j}}}{1-a^{2}} \times \sigma^{2}
$$

where $L_{j}$ depends on the scheduled transmission instants for node $j, L_{j}=$ $1,2, \ldots, n$. Thus, the estimation MSE is bounded by

$$
\underline{\varphi_{j}^{D}} \leq \varphi_{j}^{D}(t) \leq \overline{\varphi_{j}^{D}}
$$

where lower bound is given by $\varphi_{j}^{D}=\sigma^{2}$ and upper bound is given by $\overline{\varphi_{j}^{D}}=$ $\frac{1-a^{2 n}}{1-a^{2}} \times \sigma^{2}$.

From (17), (18) and (21), the minimum steady-state estimation MSE for probabilistic channel access, denoted as $\varphi_{j}^{P}(t)^{*}$, is given by

$$
\varphi_{j}^{P}(t)^{*}=\frac{\sigma^{2}}{1-a^{2}+\frac{1}{n}\left(1-\frac{1}{n}\right)^{n-1} \times a^{2}}
$$


when $n$ is sufficiently large, (33) can be approximated by

$$
\varphi_{j}^{P}(t)^{*} \rightarrow \frac{n e}{a^{2}+n e\left(1-a^{2}\right)} \times \sigma^{2}
$$

By comparing (32) and (33), numerical analysis shows that, for $0<|a|<$ 1 ,

$$
\underline{\varphi_{j}^{D}}<\varphi_{j}^{P}(t)^{*} \leq \overline{\varphi_{j}^{D}} .
$$

Therefore, when $0<|a|<1$, estimation MSE while using probabilistic design falls in the same range of that of using deterministic channel access as $t \rightarrow \infty$.

\subsection{Case $1<|a|<\infty$}

For the process (1) with bounded noise variance $\sigma^{2}>0$ and $1<|a|<\infty$, one can get (32) and (33) by going through similar derivation in previous case. Numerical analysis shows that, for the case $|a|>1$,

$$
\varphi_{j}^{P}(t)^{*}>\varphi_{j}^{D}(t)
$$

which shows that, as $t \rightarrow \infty$, using a deterministic channel access can achieve strictly lower estimation MSE than that of probabilistic design when $|a|>1$.

\section{Hybrid Channel Access}

A combined method from previous two designs may sometimes be proposed for scalability and flexibility reasons. In this section, the hybrid design bundles several nodes into one group and scheduled communication instants are given to every group fairly in a round-robin fashion. Within the same group, each node use equal broadcast probability to contend for channel access.

Assume there are $n$ nodes and $\theta$ groups, then the number of nodes in one group $m$ is given by $m=\frac{n}{\theta}$. Of course, $n, \theta, m$ must be chosen to be positive integers to make this formulation meaningful. The communication probability $p^{\theta}$ for each node in the group is given by $p^{\theta}=\frac{1}{m}$ when this group is scheduled to use the channel; otherwise, $p^{\theta}=0$. We denote the steadystate estimation MSE, defined in (6), while using the Hybrid- $\theta$ method as $\varphi_{j}^{H_{\theta}}$. 


\subsection{Case $|a|=1$}

For the process (1) with bounded noise variance $\sigma^{2}>0$ and $|a|=1$. Since nodes within the same group will contend for channel access every $\theta$ slots, the probability for receiver $i$ to get an update from node $j$ successfully, received at $k$ slots ago, can be written as

$$
P_{i j}^{\theta}\left(k=L_{j}+r \times \theta\right)=p^{\theta}\left(1-p^{\theta}\right)^{m-1} \times\left[1-p^{\theta}\left(1-p^{\theta}\right)^{m-1}\right]^{r}
$$

where $r=0,1,2, \ldots$ and $L_{j}$ depends on the group communication instants of node $j, L_{j}=1,2, \ldots, \theta$. With (35), mean inter-arrival time $E[k]$ is bounded by

$$
1-\theta+n \times\left(1-\frac{\theta}{n}\right)^{1-\frac{n}{\theta}} \leq E[k] \leq n \times\left(1-\frac{\theta}{n}\right)^{1-\frac{n}{\theta}} .
$$

As usual, (9) can be specified as similar form in (13). Therefore, the steady-state estimation MSE is bounded by

$$
\underline{\varphi_{j}^{H_{\theta}}} \leq \varphi_{j}^{H_{\theta}}(t) \leq \overline{\varphi_{j}^{H_{\theta}}}
$$

where lower bound is given by

$$
\underline{\varphi_{j}^{H_{\theta}}}=\left(1-\theta+n\left(1-\frac{\theta}{n}\right)^{1-\frac{n}{\theta}}\right) \times \sigma^{2}
$$

and upper bound is given by

$$
\overline{\varphi_{j}^{H_{\theta}}}=n\left(1-\frac{\theta}{n}\right)^{1-\frac{n}{\theta}} \times \sigma^{2}
$$

When $n$ is sufficiently large, the lower bound (37) can be approximated by

$$
\underline{\varphi_{j}^{H_{\theta}}} \rightarrow(1-\theta+n e) \times \sigma^{2}
$$

and upper bound (38) can be approximated by

$$
\overline{\varphi_{j}^{H_{\theta}}} \rightarrow n e \times \sigma^{2}
$$

If $\theta=1$, i.e. the probabilistic scheme, (37) and (38) reduce to (29). If $\theta=n$, i.e. the deterministic scheme, (36) reduces to (28).

Comparing (28), (29), and (36), numerical analysis shows that, when $|a|=1$,

$$
\varphi_{j}^{P}(t)^{*}>\varphi_{j}^{H_{\theta}}(t)>\varphi_{j}^{D}(t)
$$

which says that, deterministic channel access is the one that minimizes MSE among three methods. 


\subsection{Case $0<|a|<1$}

Now consider the process (1) with bounded noise variance $\sigma^{2}>0,0<|a|<$ 1. Plugging (35) into the same form of (20), and let $t \rightarrow \infty$, we can get the range of $E\left[a^{2 k}\right]$

$$
\underline{E\left[a^{2 k}\right]} \leq E\left[a^{2 k}\right] \leq \overline{E\left[a^{2 k}\right]}
$$

where lower bound is

$$
\underline{E\left[a^{2 k}\right]}=\frac{a^{2 \theta} \times p^{\theta}\left(1-p^{\theta}\right)^{m-1}}{1-\left(1-p^{\theta}\left(1-p^{\theta}\right)^{m-1}\right) \times a^{2 \theta}}
$$

and upper bound is

$$
\overline{E\left[a^{2 k}\right]}=\frac{a^{2} \times p^{\theta}\left(1-p^{\theta}\right)^{m-1}}{1-\left(1-p^{\theta}\left(1-p^{\theta}\right)^{m-1}\right) \times a^{2 \theta}}
$$

With (9) and (41), and the steady-state estimation MSE is bounded by

$$
\underline{\varphi_{j}^{H_{\theta}}} \leq \varphi_{j}^{H_{\theta}}(t) \leq \overline{\varphi_{j}^{H_{\theta}}}
$$

where lower bound is given by

$$
\underline{\varphi_{j}^{H_{\theta}}}=\frac{\sigma^{2}}{1-a^{2}} \times\left(1-\frac{a^{2} \times \frac{\theta}{n}\left(1-\frac{\theta}{n}\right)^{\frac{n}{\theta}-1}}{1-a^{2 \theta}+a^{2 \theta} \times \frac{\theta}{n}\left(1-\frac{\theta}{n}\right)^{\frac{n}{\theta}-1}}\right)
$$

and upper bound is given by

$$
\overline{\varphi_{j}^{H_{\theta}}}=\frac{\sigma^{2}}{1-a^{2}} \times \frac{1-a^{2 \theta}}{1-a^{2 \theta}+a^{2 \theta} \times \frac{\theta}{n}\left(1-\frac{\theta}{n}\right)^{\frac{n}{\theta}-1}}
$$

When $n$ is sufficiently large, lower bound (45) can be approximated by

$$
\underline{\varphi_{j}^{H_{\theta}}} \rightarrow \frac{\sigma^{2}}{1-a^{2}} \times\left(1-\frac{a^{2} \times \theta}{n e\left(1-a^{2 \theta}\right)+a^{2 \theta} \times \theta}\right)
$$

and upper bound (46) can be approximated by

$$
\overline{\varphi_{j}^{H_{\theta}}} \rightarrow \frac{\sigma^{2}}{1-a^{2}} \times \frac{n e\left(1-a^{2 \theta}\right)}{n e\left(1-a^{2 \theta}\right)+a^{2 \theta} \times \theta}
$$


Similar to previous case, if $\theta=1$, i.e. the probabilistic scheme, (45) and (46) reduce to (33). If $\theta=n$, i.e. the deterministic scheme, (44) reduces to (32).

Now comparing (32), (33) and (44), numerical analysis shows that, for the case $0<|a|<1$,

$$
\underline{\varphi_{j}^{D}}<\underline{\varphi_{j}^{H_{\theta}}} \leq \varphi_{j}^{P}(t)^{*} \leq \overline{\varphi_{j}^{H_{\theta}}} \leq \overline{\varphi_{j}^{D}}
$$

which shows that, as $t \rightarrow \infty$, three channel access designs have estimation MSE roughly within the same range.

\subsection{Case $1<|a|<\infty$}

Now consider the process (1) with bounded noise variance $\sigma^{2}>0,1<|a|<$ $\infty$. One may go through similar derivation as previous case to get (44), (45) and (46). But note that, similar to (26), those results are valid only when

$$
\left(1-p^{\theta}\left(1-p^{\theta}\right)^{m-1}\right) \times a^{2 \theta}<1 .
$$

In other words, to have bounded estimation MSE for hybrid design, parameters $a, n, \theta$ must satisfy below condition,

$$
|a|<\left(1-\frac{\theta}{n}\left(1-\frac{\theta}{n}\right)^{\frac{n}{\theta}-1}\right)^{-\frac{1}{2 \theta}}
$$

where $1 \leq \theta<n$. (49) gives a relaxed condition than the limitation of probabilistic design given in (27). In Hybrid- $\theta$ channel access, the contention from all nodes, as what happens in probabilistic design, has been distributed to several small scale contentions within a group.

Comparing (32), (33) and (44), numerical analysis shows that, for the case $|a|>1$,

$$
\varphi_{j}^{P}(t)^{*}>\varphi_{j}^{H_{\theta}}(t)>\varphi_{j}^{D}(t) .
$$

That is, deterministic channel access can achieve strictly lower estimation MSE as $t \rightarrow \infty$.

\section{Summary and Future Work}

Model-based estimation over the multi-access network is studied in this report. A mathematical framework is proposed and the estimation MSE of 
three channel access schemes is analyzed. Asymptotic behavior and stability condition are also derived. Our results suggest that, while designing NCSs, the channel access scheme should be chosen based on $|a|$ of the dynamic process (1). If $|a| \geq 1$, the deterministic design (round-robin scheduling) can achieve strictly lower estimation MSE. Otherwise, for $0<|a|<1$, three methods have roughly the same range of MSE as $t \rightarrow \infty$.

Future work includes the generalization from current analysis to more practical applications, e.g. vehicle's active safety mechanism mentioned in introduction. Meanwhile, [13] proves that, for a sender-receiver pair, optimal communication design should incorporate estimation error and such correlation can help improve estimation accuracy. We also want to explore the optimal communication design for estimation in a multi-access channel setting.

\section{References}

[1] D. Bertsekas, R. Gallager, Data Networks, Prentice Hall, 1992.

[2] T. M. Cover, J. A. Thomas, Elements of Information Theory, second edition, Telecommunications, New York: Wiley, 1991.

[3] S. Tatikonda, A. Sahai, S. K. Mitter, "Stochastic linear control over a communication channel," IEEE Trans. on Automat. Contr., vol.49, no.9, Sept. 2004.

[4] P. Seiler, R. Sengupta, "An $\mathrm{H}_{\infty}$ approach to networked control," IEEE Trans. on Automat. Contr., vol.50, no.3, March 2005.

[5] X. Lu, J.K. Hedrick, M. Drew, "ACC/CACC-control design, stability and robust performance," Proc. of the Amer. Contr. Conf., May 2002.

[6] N. Elia, S. Mitter, "Stabilization of linear systems with limited information," IEEE Trans. on Automat. Contr., vol.46, no.9, 2001.

[7] W. S. Wong, R. W. Brockett, "Systems with finite communication bandwidth constraints. II: Stabilization with limited information feedback," IEEE Trans. on Automat. Contr., vol.44, no.5, 1999. 
[8] J.K. Yook, et. al., "Trading computation for bandwidth: Reducing communication in distributed control systems using state estimators," IEEE Trans. Contr. Syst. Technol, vol.10, no.4, July 2002.

[9] G.N. Nair, R.J. Evans, "Exponential stabilisability of finite dimensional linear systems with limited data rates," Automatica, vol.39, no.4, Apr. 2003.

[10] Y. Xu, J. Hespanha, "Communication Logics for Networked Control Systems," Proc. of the Amer. Contr. Conf., June 2004.

[11] B. Sinopoli, et. al., "Kalman Filtering with Intermittent Observations," IEEE Trans. Automat. Contr., vol.49, no.9, Sept. 2004.

[12] X. Liu, A. Goldsmith, "Kalman filtering with partial observation losses," Proc. of the IEEE Conf. Decision and Contr., Dec. 2004.

[13] Y. Xu, J. Hespanha, "Optimal Communication Logics for Networked Control Systems," Proc. of the IEEE Conf. Decision and Contr., Dec. 2004.

[14] Y. Xu, J. Hespanha, "Estimation under uncontrolled and controlled communication in Networked Control Systems," Proc. of the IEEE Conf. Decision and Contr., Dec. 2005.

[15] Z. Jin, V. Gupta, R. M. Murray, "State Estimation over Packet Dropping Networks using Multiple Description Coding," Automatica, vol.42, no.9, Sept. 2006.

[16] D.R. Cox, "Some statistical methods connected with series of events," Jour. Royal Stat. Society, vol.17, no.2, 1955.

[17] A. Sahai, "Anytime Information Theory," Ph.D. dissertation, Dept. EECS, MIT, Cambridge, MA, Feb. 2001.

[18] C. Chang, A. Sahai, "Sequential Random Coding Error Exponents for Multiple Access Channel," IEEE WirelessCom 05 Symposium on Information Theory, Feb. 2005.

[19] J. Hespanha, et. al., "A Survey of Recent Results in Networked Control Systems," Proc. of IEEE, Special Issue on Networked Control Systems, Jan. 2007. 